\title{
From Microjoining to Nanojoining
}

\author{
Y. Zhou ${ }^{*}$ and A. Hu \\ Centre for Advanced Materials Joining, University of Waterloo, 200 University Avenue West, Waterloo, Ontario, N2L \\ 3G1, Canada
}

\begin{abstract}
A review is provided of the recent advances and challenges in nanojoining, as compared to microjoining and even regular joining processes. Nanojoining examples are given and analyzed in various joining categories such as solidstate bonding, soldering/brazing and fusion welding. Proposed strategies for nanoassembly and nanojoining, e.g., selforganization and inkjet printing, are also highlighted.
\end{abstract}

Keywords: Nanojoining, microjoining, solid-state nanobonding, nanosoldering, nanobrazing, fusion nanowelding.

\section{INTRODUCTION}

Joining (welding, brazing, soldering, bonding, etc.), whether at macro-, micro- or nano-scale, is an essential step of man-made product manufacturing and assembly, providing mechanical support and integration, electrical connection, optical coupling, environmental protection, etc. [1] This review focuses only on joining processes that aim to produce permanent unions between macro-, micro- and nano-sized building blocks to be assembled, through the formation of primary (and occasionally secondary) chemical bonds between the faying surfaces. The exception to this metallurgical definition is mechanical joining (fastening, riveting, crimping, etc.), in which the original free surfaces of the parts are retained mostly unaltered in mechanical joints. The continuing miniaturization of engineered devices and systems, coupled with ever-growing demands for cost reduction, enhanced performance and reliability, etc., brings new challenges to the technology of joining.

The term "microjoining" is already popular in industries while "nanojoining" is relatively new, used mostly in research and development communities, although both terms are very loosely defined and often used relative to conventional (regular, large- or macro-scale) welding and joining. One common way of defining "microjoining" or "nanojoining" is based on the dimensions of the parts or building blocks to be joined. For example, joining of parts with a characteristic dimension, eg, sheet thickness or wire diameter, up to a few hundred nanometers could be called nanojoining. Similarly, microjoining would deal with parts with characteristic dimension smaller than a few hundred micrometers but larger than hundreds of nanometers [1]. But this form of definition could be too strict and sometimes misleading. In fact, there are other potentially more effective ways to define microjoining and nanojoining, such as based on industrial/application areas, process characteristics, equipment characteristics (e.g., the precision of such a

*Address correspondence to this author at the Centre for Advanced Materials Joining, University of Waterloo, 200 University Avenue West, Waterloo, Ontario, N2L 3G1, Canada; Tel: 519-8884567-36095;

E-mail:nzhou@uwaterloo.ca system), and/or the materials to be joined. For example, resistance microwelding is mostly used to join non-ferrous materials in the fabrication of precision components and devices with sheet thickness or wire diameter in an approximate range from 20 to $400 \mu \mathrm{m}$, such as in sensors, actuators and medical devices, using electrode forces in the range of 1-700 N. This is in comparison to regular resistance welding, predominantly used in joining steels or aluminum alloys as in autobody and appliance assembly with sheet thickness or wire diameter usually above $0.5 \mathrm{~mm}$ and electrode forces above a few hundred kilonewtons $(\mathrm{kN})$ [1]. Microelectronic wire bonding is an ultrasonic microjoining process used for semiconductor chip-level interconnections using wire diameters typically less than 25 microns but occasionally at a few hundred microns for power electronics applications, which compares to (regular) ultrasonic welding for structural applications that deals with wire diameter or sheet thickness typically above a few hundred microns [1].

Many microjoining or microwelding processes can trace their origins back to the 1950 s while most nanojoining processes were only developed in the past decade. For example, a capacitor discharge machine was introduced then for resistance welding of orthodontic appliances, and this development was later adopted in the aerospace industry in the 1960s [2]. Thermocompression wire bonding, a variant of hot pressure welding, was developed by Bell Laboratories in 1957 [3] and later evolved into ultrasonic wire bonding in the 1960s and thermosonic wire bonding, a combination of thermocompression and ultrasonic wire bonding, in 1970 [4]. The wire bonding processes have been one of the key chiplevel interconnection technologies in the success of microelectronic products. While wire bonding was intended for microjoining applications, electron beam welding, developed in 1958 [5], is suitable for macro-, micro- and even nano-scale joining because of the precise beam quality, and fixturing and controls integrated with the system.

Although effective microjoining has been an integral part of manufacturing for many decades in microelectronics, medical, aerospace, and defence industries, microjoining continues to face challenges because of ever advancing miniaturization. For example, novel microjoining processes are urgently needed for packaging and interconnecting in 
MEMS (MicroElectroMechanical Systems), also called microsystems or micromachines, where individual electrical, mechanical, fluidic, and optical components need to be connected and coupled to the macroscopic external environment [6]. The latter causes a particular problem in sensor technology where, on one hand, systems must be protected from mechanical damage and corrosion, while on the other hand, they must be exposed as intimately as possible to the environment to obtain true, undistorted physical and chemical values [6]. For this reason, "packaging and interconnecting techniques will play the key role in any further industrial expansion (of microsystems)" [6].

Further down the road of miniaturization to nanoscale, after many years of significant progress in nanoscience and nanotechnology such as in materials development and testing, there is an emerging need to join nanoscale building blocks, such as nanowires and nanotubes, to themselves to form nanoscale devices and systems, and then to join these to the surroundings, to be integrated into micro- and macroscale devices and systems. Otherwise, industrial scale production of these nanoscale devices and systems is not possible, as experienced both at macro- and micro-scales. In this paper, recent advances in nanojoining, as compared to microjoining, are reviewed, and process characteristics and technical challenges are also analyzed with selected examples.

\section{MECHANISMS AND CLASSIFICATIONS OF JOINING}

In principle, two ideal solid surfaces, e.g., both perfectly clean and atomically flat, will join together if brought into intimate contact, because they will be drawn together spontaneously by interatomic forces $[1,7]$. The driving force for this joining is the reduction of Gibbs free energy of the system by eliminating, reducing or replacing high-energy free surfaces by low-energy interfaces. This theoretical principle has been confirmed in tightly controlled environments, such as in direct silicon wafer bonding [8]. However, most engineering surfaces are characterized as rough and contaminated with oxides, etc. (especially as seen in most macro- and even micro-joining operations), requiring some form of energy, usually heat and/or pressure, to be applied to overcome these surface impediments to make a joint. Roughly, there are four major types of joining processes based on the physical states of the parts to be joined: solid-state bonding, soldering/brazing, fusion welding, and adhesive bonding. This classification has been mainly developed for metals although some of concepts can be also used for other materials such as ceramics and plastics.

Solid-state bonding is mainly achieved by pressure where no melting occurs but solid-state diffusion can assist/promote the joint formation. Fusion welds and brazed/soldered joints are achieved by melting and epitaxial solidification of molten metal, but localized melting of base metals occurs in fusion welding, while only the melting of filler metals occurs in brazing/soldering (in initial stages at least). While the melting points in brazing are above $450^{\circ} \mathrm{C}$, those in soldering are below $450^{\circ} \mathrm{C}$. If liquid is removed during the process, solid-state joints can also be produced in fusion welding, soldering and brazing processes, e.g., as in resistance cross-wire microwelding [9]. In adhesive bonding, organic (and sometime inorganic) adhesives are used to join parts together. Adhesive bonding, soldering and brazing can be easily applicable to dissimilar material combinations if the adhesives, solders or brazes are compatible with both sides, while interlayers may be needed in solid-state bonding of dissimilar material parts. It is expected that, besides the surface impediments (roughness and contamination), other challenges arise in micro- and especially nano-joining because of continuing miniaturization and the associated laws of physics, e.g., significantly increased surface areas and reduced volumes. These would for example cause difficulties in manipulation of the building blocks and in avoidance of damage to their internal structure and properties as discussed later in this paper.

Microjoining processes can be still grouped according to the traditional classification method, in terms of solid-state bonding, fusion welding, brazing/soldering and adhesive bonding (see examples listed in Table 1) [1]. But whether this classification can be applied to nanojoining is still a question to be answered. Most nanojoining processes developed so far, especially those for joining metallic nanomaterials, can fit well into Table 1. One example of the processes in doubt is the direct joining of carbon nanotubes (CNTs) using a focused electron beam [10]. In this process, the electron beam is directed to induce structural defects, "vacancies" and "interstitials", at the crossing point of two CNTs. Self rearrangement of those affected carbon atoms occurs at high specimen temperature $\left(800^{\circ} \mathrm{C}\right.$ in Ref. [10]) so as to form heptagonal or octagonal rings thus producing inter-nanotube junctions, as shown in Fig. (1). The lowering of energy of the system is obviously the driving force to form the junctions, confirmed by molecular dynamics simulation as shown in Fig. (2). One could argue that this direct joining of CNTs can be grouped as a solid-state bonding process since no melting occurs (Table 1).

While new processes will be continuously invented, many existing microjoining (or even macrojoining/regular joining) processes may be also further developed and modified for the purpose of nanojoining. For example, electron beam welding, a process well established for macroand micro-applications $[1,11]$ and also used for CNT joining [10], should be very promising for joining of nanoscale metal parts because of its beam quality.

\section{SOLID-STATE NANOBONDING}

Diffusion nanobonding using metallic nanoparticles as bond layers is a popular nanojoining process currently under study. In this solid-state nanobonding process, nanoparticles are sintered to form networks and at the same time these networks are joined to substrates, all assisted by diffusion with driving force to reduce surface areas. As the size of nanoparticles reduces, diffusion is enhanced because of lower activation energy and increased specific surface energy, which can result in the decrease of sintering and bonding temperature [26]. This is important since there is increasing interest in development of low temperature joining processes for polymeric based microelectronics applications [12]. 
Table 1. Typical Microjoining and Nanojoining Processes

\begin{tabular}{|c|c|c|}
\hline & Microjoining [1] & Nanojoining \\
\hline Solid-state bonding & $\begin{array}{l}\text { Anodic/Wafer, Diffusion, Ultrasonic wire bonding; Cold, Explosive, } \\
\text { Friction stir, Friction welding, etc. }\end{array}$ & $\begin{array}{c}\text { Electron beam welding* [10], Diffusion bonding }[12,13] \text {, } \\
\text { Ultrasonic welding [14, 15], Cold welding [16] }\end{array}$ \\
\hline Soldering/Brazing & $\begin{array}{l}\text { Diffusion, Furnace, Induction, Laser reflow, Resistance, Eutectic } \\
\text { soldering/brazing; Dip, Wave, Vapour phase, Fluxless soldering; } \\
\text { Active brazing; Flip chip bonding; etc. }\end{array}$ & $\begin{array}{l}\text { Liquid-phase reflow soldering [17], Resistance soldering } \\
\text { [18], Active brazing [19, 20], Laser brazing [21] }\end{array}$ \\
\hline Fusion welding & $\begin{array}{l}\text { Electron \& Laser beam, Percussive, Plasma, Gas tungsten, } \\
\text { Resistance welding; Glass sealing; etc. }\end{array}$ & Laser beam welding [22, 23], Resistance welding [24] \\
\hline Adhesive bonding & $\begin{array}{l}\text { Adhesive die attachment, Adhesive flip chip bonding, Adhesive } \\
\text { sealing, etc. }\end{array}$ & Adhesive bonding [25] \\
\hline
\end{tabular}

*Note: please refer to the text for the discussion of direct electron beam welding of CNTs.
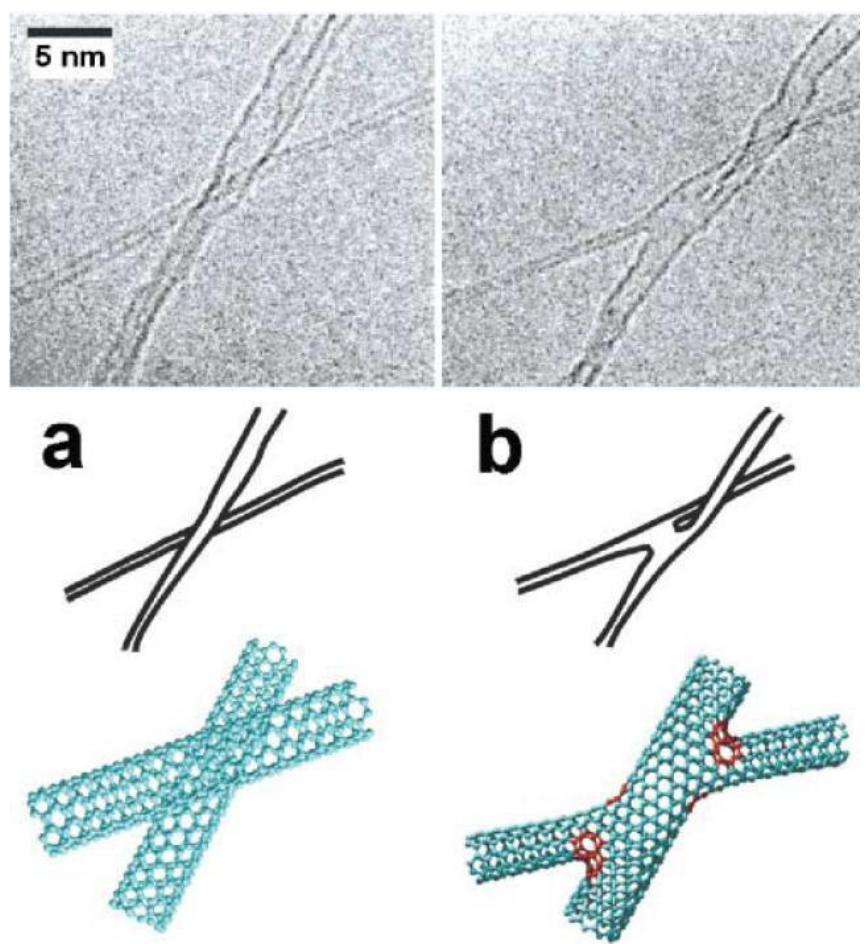

Fig. (1). (a) A single wall CNT of $\sim 2.0 \mathrm{~nm}$ in diameter crossing with another single wall CNT of $\sim 0.9 \mathrm{~nm}$ in diameter. (b) $60 \mathrm{sec}$ of electron irradiation promotes a molecular connection between two tubes, forming an " $\mathrm{X}$ " junction. Schematics show that this junction is twisted out of the plane. Molecular models of each image are provided; heptagonal rings are indicated in red [10].

In principle, bonding temperature can be reduced all the way down to room temperature, e.g., it has been reported that Ag nanoparticles were successfully sintered at room temperature [27]. However, surface coatings are needed to prevent nanoparticle self-coalescence at room temperature in long-term storage and handling before bonding. For example, a novel process has been developed to join $\mathrm{Cu}$ discs using Ag metallo-organic nanoparticles, in which the nanoparticles are covered by an organic shell to prevent selfcohesion before bonding [28]. The sintering between the nanoparticles occurs at temperatures up to $300^{\circ} \mathrm{C}$ after the decomposition of the shell. The shell decomposition, by nature an oxidation reaction, also removes the oxide film on the $\mathrm{Cu}$ surface $(\sim 10 \mathrm{~nm}$ in thickness) and makes it possible to bond the nanopartcles to the $\mathrm{Cu}$ substrates. The bonding

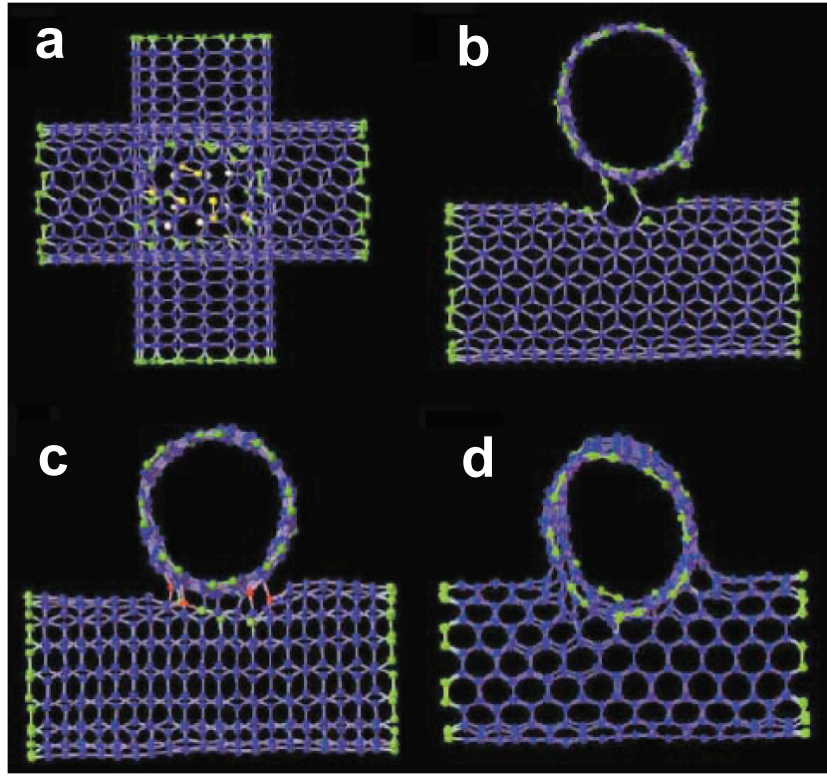

Fig. (2). Tight binding molecular dynamics (TBMD) simulated sequences of merging between two crossing $(8,8)$ CNT $(\sim 1.1 \mathrm{~nm}$ in diameter) into a unique $\mathrm{X}$ junction. (a) The simulation starts with the random creation of 20 vacancies in the lattices of two tubes in the localized neighboring region (top view). (b) After $10 \mathrm{ps}$, two links between the two defective carbon structures are formed via carbon chains (side view). (c) After $100 \mathrm{ps,} \mathrm{the} \mathrm{connection} \mathrm{between}$ the two tubes is established, although some sp3-carbon atoms (red) and dangling bonds (green) are still remaining. (d) After 220 ps, surface reconstruction occurs and the carbon system approaches an $\mathrm{X}$ junction. The reconstructed surface contains six heptagons, one octagon, one pentagon, and two dangling bonds [10].

temperature can be further reduced from 300 to $240^{\circ} \mathrm{C}$ if $\mathrm{Ag}_{2} \mathrm{CO}_{3}$, as a source of oxygen, is mixed with the $\mathrm{Ag}$ metallo-organic nanoparticles [29]. Recently, an organic shell of a mixture of citrate and $\mathrm{Ag}_{2} \mathrm{CO}_{3}$ at 1-3 nm in thickness has been developed as coating on pure $\mathrm{Ag}$ nanoparticles, which can decompose at much lower temperature [12, 13]. Fig. (3) shows scanning electron microscopy (SEM) images of condensed and sintered $\mathrm{Ag}$ nanoparticles at room temperature and $160^{\circ} \mathrm{C}$ for 10 minutes, respectively. It is evident that at room temperature these nanoparticles are well separated and at $160^{\circ} \mathrm{C}$ they start to form necks between neighbouring particles. The lowest processing temperature is found to be at about $100^{\circ} \mathrm{C}$ in 
bonding $\mathrm{Cu}$ wires to $\mathrm{Cu}$ foils, which is very promising for flexible electronics packaging on plastic substrates $[12,13]$. Alternatively, Ag nanoparticles can be in situ synthesized from the reduction of micro-scale $\mathrm{Ag}_{2} \mathrm{O}$ particles with organic solvents, such as myristyl alcohol $\left(\mathrm{C}_{14} \mathrm{H}_{24} \mathrm{OH}\right)$ [30], and used as bonding filler metals.

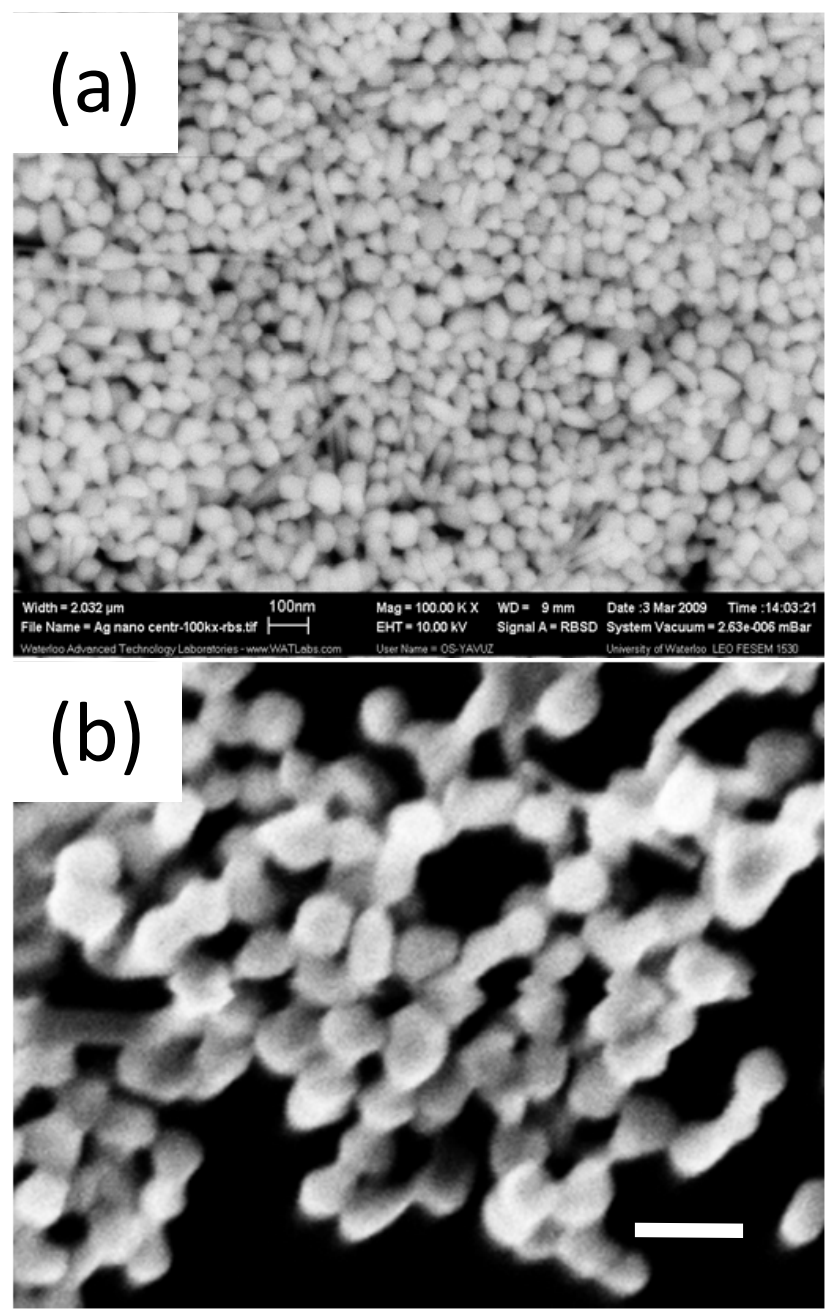

Fig. (3). SEM micrograph of sintered Ag nanoparticles at (a) room temperature and (b) $250^{\circ} \mathrm{C}$ with the scale corresponding to $100 \mathrm{~nm}$ [12].
On the other hand, diffusion bonded joints using metallic nanopartciles can also work at higher temperatures than the joining temperatures since sintered networks have higher melting points, important in applications such as automobile engines $[12,13]$.

While ultrasonic welding is commonly used in regular joining and microjoining, ultrasonic nanowelding has been developed to join CNTs to Ti electrodes, as shown in Fig. (4) [14] for the development of carbon nanotube-based photovoltaic cells with high energy conversion efficiency [15]. Low Ohmic contact has been achieved between the Ti electrodes and CNTs because of the formation of covalent CTi bonds, which is in fact the mechanism responsible for joining [14]. This was also shown by comparing the resistance across the electrodes, and by X-ray Photoelectron Spectroscopy (XPS) spectra; the resistance dropped greatly when ultrasonic power was high enough to form Ti-C covalent bonds, as shown in Fig. (5). Mechanical clamping (when the ultrasonic power was zero in Fig. (5)) was not sufficient to produce Ohmic contacts between the CNTs and Ti electrodes.

Cold nanowelding has been used to join ultrathin singlecrystal Au nanowires using only mechanical contact at close to room temperature in a high-resolution transmission electron microscope (TEM) equipped with scanning tunnelling microscope (STM) [16]. The joint strength reached $\sim 600 \mathrm{MPa}$, comparable to that of the original nanowire. The estimated applied pressure was only $\sim 4.7$ $\mathrm{MPa}$, less than $5-10 \%$ of that required for cold welding of bulk metals such as $\mathrm{Al}$ and $\mathrm{Cu}$ of a few hundred $\mathrm{mm}^{2}$ crosssectional area [31]. The difference is due not only to the much more significant influence of the surface impediments (roughness and oxides) but also the difficulties in realignment of crystallographic orientation across the bondline in regular cold welding (see detailed discussions on the mechanisms of solid-state bonding in Ref [1]). It has been indeed observed that cold nanowelding occurred more easily between two nanowires with the same growth orientation [16].

\section{NANOSOLDERING AND NANOBRAZING}

In nanosoldering or nanobrazing, molten solder or braze needs to be able to wet the parts to be joined. For a successful nanosoldering or nanobrazing, a tiny amount of (a)

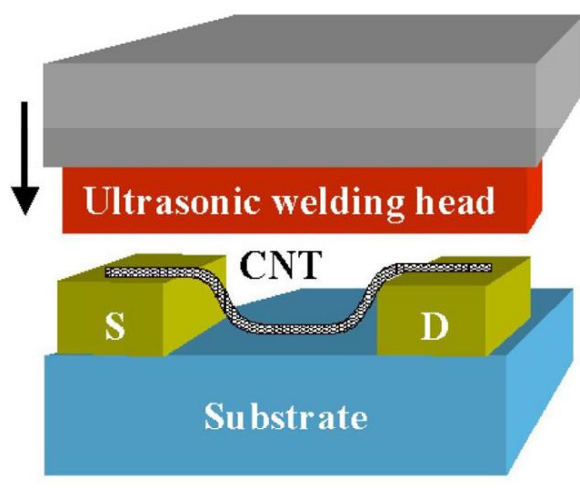

(b)

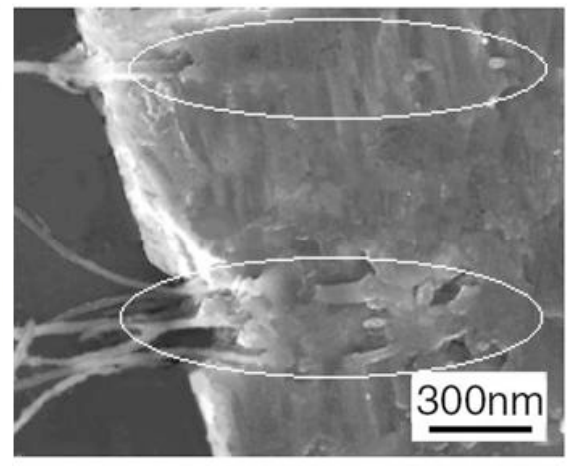

Fig. (4). (a) Schematic of the setup for ultrasonic welding with a clamping force (indicated by the arrow) to press the welding head against the nanotube, and Ti electrodes (S and D) and (b) SEM image of multiple CNTs welded on the Ti electrodes [14]. 


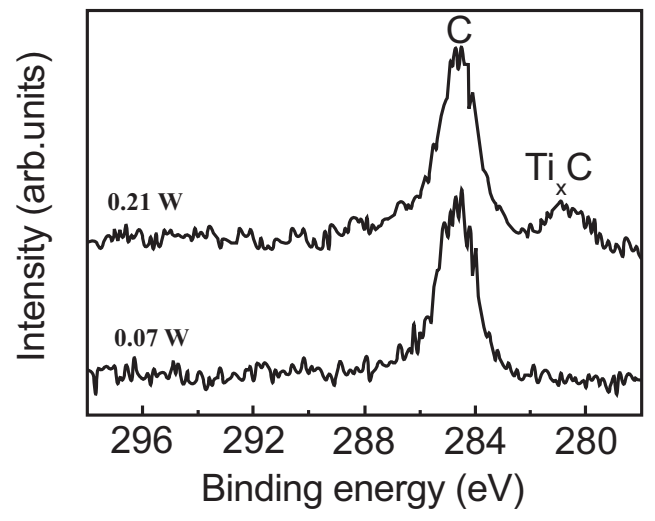

(a)

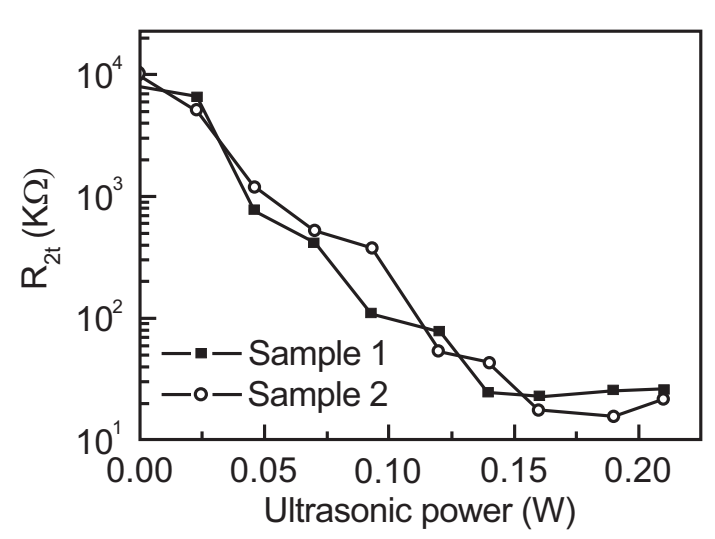

(b)

Fig. (5). (a) XPS spectra of the weld zone at ultrasonic power of $0.07 \mathrm{~W}$ and $0.21 \mathrm{~W}$, and (b) Two-terminal (2t-) resistances across the electrodes as a function of ultrasonic power. Before ultrasonic nanowelding, the 2t-resistances of sample 1 and 2 were 49.4 and $55.8 \mathrm{M} \Omega$, respectively; the ultrasonic power of $0 \mathrm{~W}$ in the figure represents that the ends of the CNT were pressed against the electrodes by pure clamping force without ultrasonic power applied [14].

solder also has to be precisely delivered to the bonding area; and thus, various processing and manipulation strategies need to be developed, especially for mass production [1].

Recently nanosoldering has drawn significant attention as a possibly significant joining process in the assembly and integration of nanoelectronics devices [32]. Girit and Zettel [33] used a micromanipulator with a tungsten tip to solder In-Tin alloy to make an ohmic contact on graphene, a single atomic layer of carbon. With a nanomanipulator within a $\mathrm{SEM}$, Peng et al. joined Au to Pt-Co nanowires with $\mathrm{SnAu}$ nanowires as solders melted by Joule heating [18]. This kind of process, where only a single weld is made, can be slow and costly because of the requirements for accurate manipulation of the parts and execution of the joining operation.

As an attractive alternative, batch operation allows multiple joints made at the same time. This is illustrated in the following example on reflow soldering of multisegmented metal nanowires $[17,32,34,35]$. Multisegmented metal (such as $\mathrm{Sn} / \mathrm{Au} / \mathrm{Ni}$ ) nanowires were fabricated using an electrodeposition method in nanoporous templates $[32,17,34,35]$. In one example, Tin was grown on the ends of $\mathrm{Au}$ nanowires with $\mathrm{Ni} / \mathrm{Au}$ as under-layers $(\mathrm{Ni}$ as a diffusion barrier and $\mathrm{Au}$ as wetting layer) and then the $\mathrm{Sn} / \mathrm{Au} / \mathrm{Ni}$ coated $\mathrm{Au}$ nanowires were reflow soldered together in a liquid medium [35]. A magnetic field was also employed to align the nanowires to form three-dimensional nanowire networks [36].

Mafune et al. [21] used nanosecond pulsed laser to melt $\mathrm{Au}$ nanoparticles to braze Pt nanoparticles together to form three-dimensional networks, shown in Fig. (6). Wu et al. [19] have brazed CNT bundles to Ni electrodes with Ag-Cu$\mathrm{Ti}$ alloy under vacuum of $10^{-6}$ Torr. XPS analysis revealed the brazing mechanism as the formation of Ti-C covalent bonds [19], similar to what happened in ultrasonic welding of CNTs to Ti electrodes $[14,15]$. It is well known that an active alloying element, such as $\mathrm{Ti}$, is needed so that molten braze or solder will be able to wet on ceramics in so-called active brazing/soldering [1]. The wetting of $\mathrm{Ag}-\mathrm{Cu}-\mathrm{Ti}$ alloy on CNT is shown in Fig. (7) [19]. The joint strength displayed a maximum around $900^{\circ} \mathrm{C}$ to $950^{\circ} \mathrm{C}$ because at higher temperatures CNTs started to degrade due to their reaction with the brazing materials and catalytic compounds [19]. With the brazed CNT bundles an incandescent lamp filament can be fabricated with high light emission efficiency due to the much lower contact resistance enabled by active brazing, shown in Fig. (8) [20].

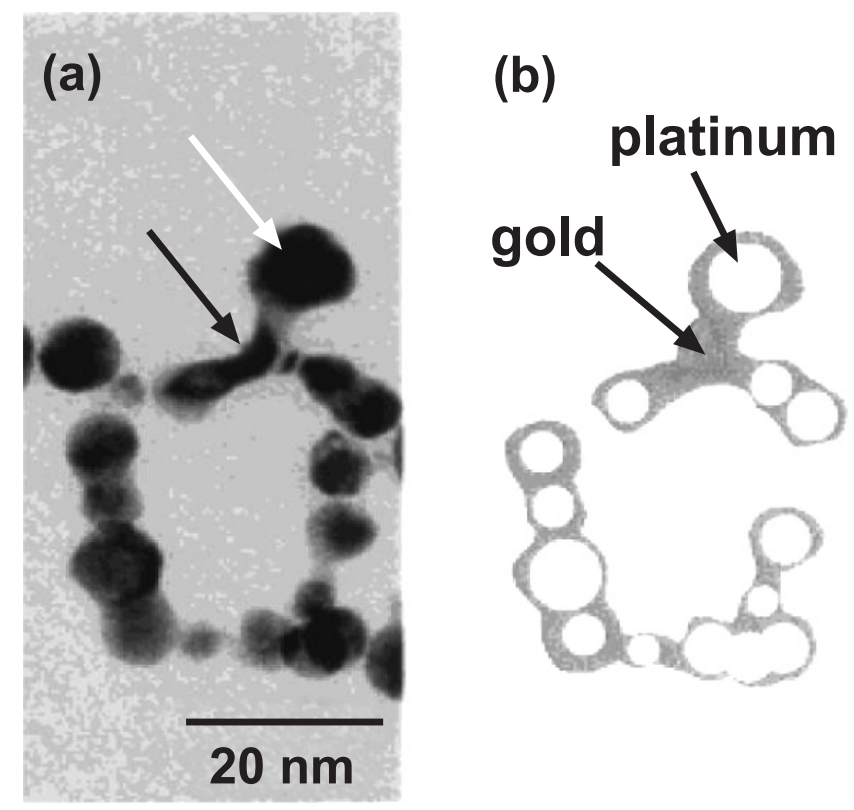

Fig. (6). (a) Electron micrograph of Pt and Au networks formed by laser brazing and (b) schematic showing how Pt nanoparticles were held together by molten $\mathrm{Au}$ [21].

\section{FUSION NANOWELDING}

Fusion nanowelding processes have been attempted with various heat sources, such as laser beam [22, 23], Joule heating [24, 37] similar to that used in resistance welding [1], and even a resistively heated hot stage [38]. Fig. (9) shows the welding of $\mathrm{Pt}$ nanowires, in which two $\mathrm{Pt}$ nanowires are touched end-to-end by piezoelectric manipulation inside a SEM chamber followed by passage of 
a welding current [37]. The joint is formed by resolidification (fusion) of the molten metal at the faying surfaces produced by Joule heating. The voltage across the junction reduces slightly once the weld forms and then stays unchanged, indicating an Ohmic contact has been formed. This resistance nanowelding approach has also been used to join dissimilar metals, e.g., Pt nanowire to thin $\mathrm{Au}$ wire to produce heterojunctions as shown in Fig. (10) [24].

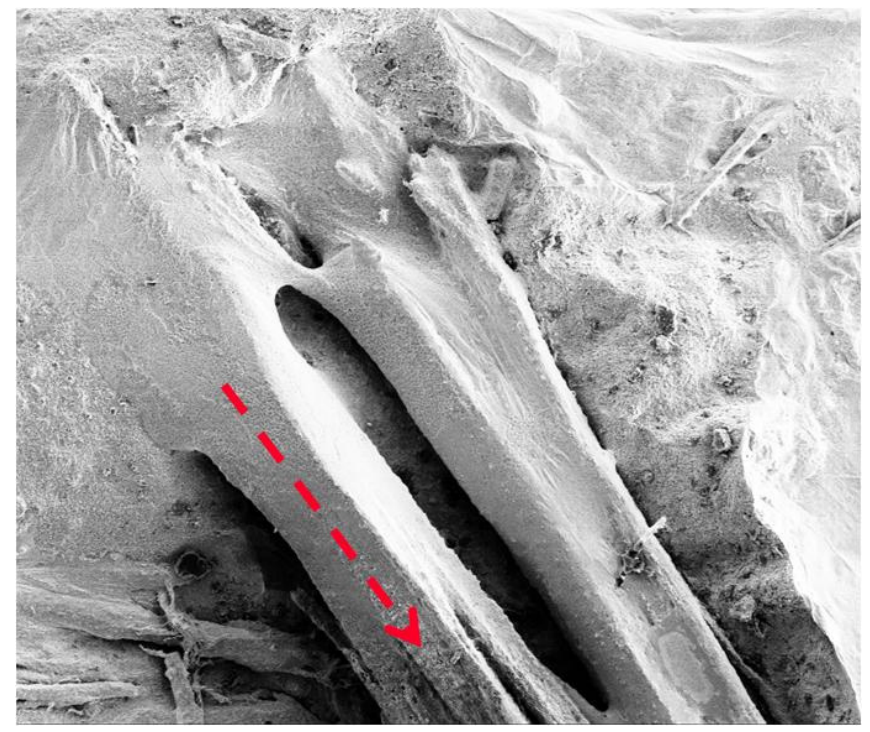

Fig. (7). SEM image showing the brazing alloy Ag-Cu-Ti wetted on the CNT bundles [19].

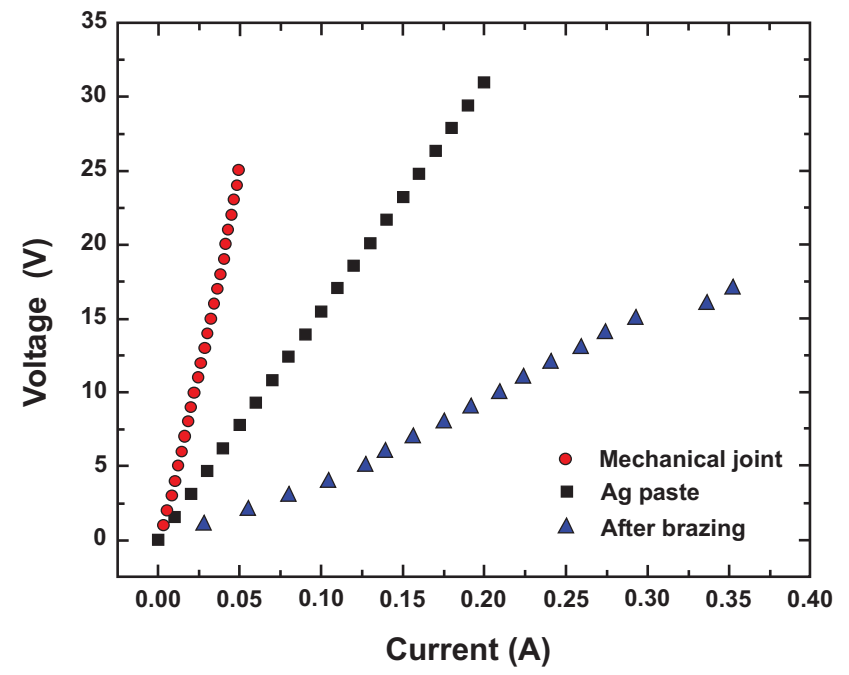

Fig. (8). Voltage-current curves of various Ni-CNT-Ni joints as made by mechanical clamping, Ag-paste adhesive bonding and Ag$\mathrm{Cu}$-Ti active brazing [20].

A hot stage within a SEM has been used to weld two crossed semiconductor Ge nanowires coated with thin layers of carbon sheath [38]. This localized welding is possible due to the porous nature of the carbon sheath, which allows the molten Ge to fuse together and later solidify at the contact point.

The fusion nanowelding methods with manipulation and observation in SEM, STM and TEM display remarkable success in joining nanometals. But these methods, fall in the category of "single weld" operations, and as such suffer from various issues, such as slow processing time and high equipment cost, which make them less attractive in mass production.

\subsection{Ultrafast Laser Nanowelding}

Fusion nanowelding by ultrashort laser pulse (shorter than picoseconds) is reviewed here as conceptually separated from other fusion nanowelding processes since the nature of interaction between ultrafast laser pulses and materials is known as non-thermal processing [39]. As the electronlattice thermal coupling time (typical a few picoseconds) is much longer than the laser pulse width, the electrons do not have enough time to transfer energy to the lattice. Electrons are excited, ejected and thereby weaken the chemical bonds of lattice atoms, resulting in "melting" of surface atoms, which is a non-thermal phenomenon [39, 40]. This melting also occurs only on the surface to a nanoscale depth without damaging the bulk and thus can be used for welding of nanoparticles [22]. Fig. (11) shows Au nanoparticles welded with 100 fs laser pulses [22].

\section{ADHESIVE BONDING}

Adhesive bonding, suitable for joining at different levels: regular to micro- and nano-scales, uses an organic (or sometime inorganic) substance (adhesive) to join two parts together [1]. Similar to soldering/brazing, liquid adhesive needs to be able to wet the surfaces of the building blocks so that the free energy of the system associated with the original surfaces is reduced [1]. Gu et al. [40] has used adhesives to join multisegmented $\mathrm{Au}-\mathrm{Ni}-\mathrm{Au}$ rods of 150-250 $\mathrm{nm}$ diameter and 2-6 microns length into two- or three-dimensional structures/networks. Good wetting of the adhesive on the nanorods is clearly shown in Fig. (12) [25].

\section{NANOASSEMBLY}

Manipulating and positioning of nanoscale building blocks are the first steps of any successful nanojoining. Besides the operation with the help of SEM, AFM, STM and TEM, other manipulating and positioning methods, such as self-organization, nanoink imprinting, near-field optical microscopy and optical tweezers are emerging. These nanoscopic manipulation strategies make the mass fabrication of nanodevices feasible in combination with different nanojoining processes.

Self-organization, which allows hundreds and thousands of building blocks aligning in desired directions to form ordered one-, two- or three-dimensional structures, is found at various scales, spanning from nano-, micro-, millimetresize, even to universal systems [41]. Such an assembly process is simple, fast, scalable for batch processing, and cost-effective. Recent progress in self-organization has been achieved through many strategies such as shape recognition [42], capillary-force [43], molten solder [44], and magnetic field [45]. Fig. (13) shows reflow soldering of magnetically self-organized $\mathrm{Sn}-\mathrm{Au}-\mathrm{Ni}-\mathrm{Au}-\mathrm{Sn}$ multisegmented nanowires in a liquid medium $[17,36]$.

Nanoink imprinting is another strategy gaining interest because it is an economical and highly functional technology for microscale patterning of metallic nanoparticle traces [4648]. It employs piezo-electronically driven micro-nozzles to directly write the patterns by printing the nanoparticle ink, 

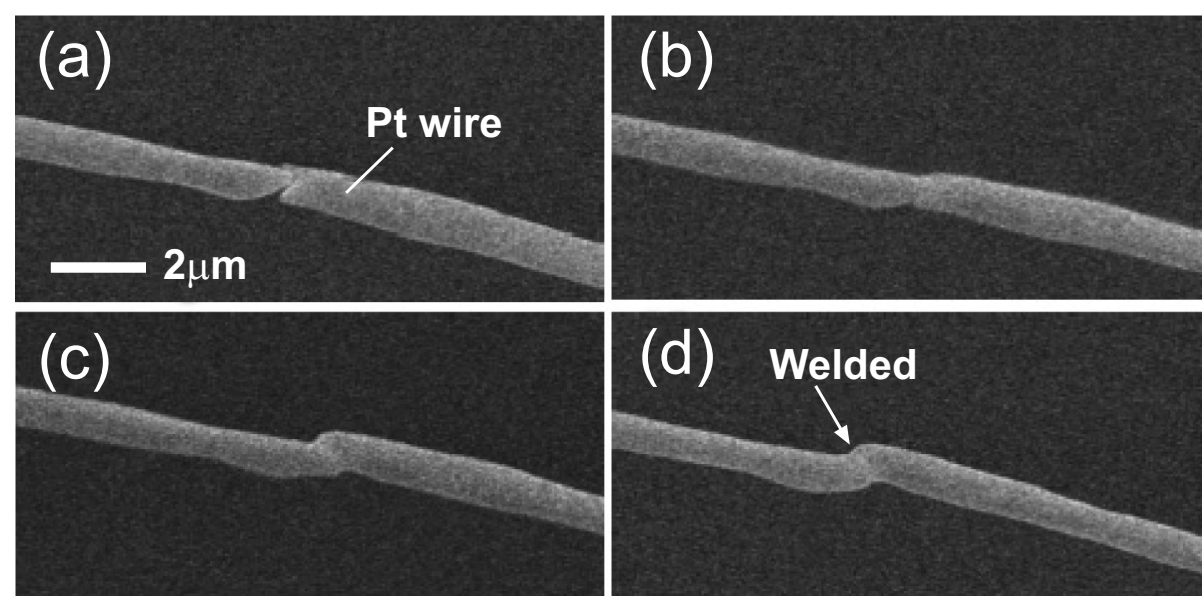

Fig. (9). SEM micrographs of welding of Pt nanowires: (a) before current supply, (b-d) the snapshots at 1, 6, and $21 \mathrm{~s}$ after starting the current [37].
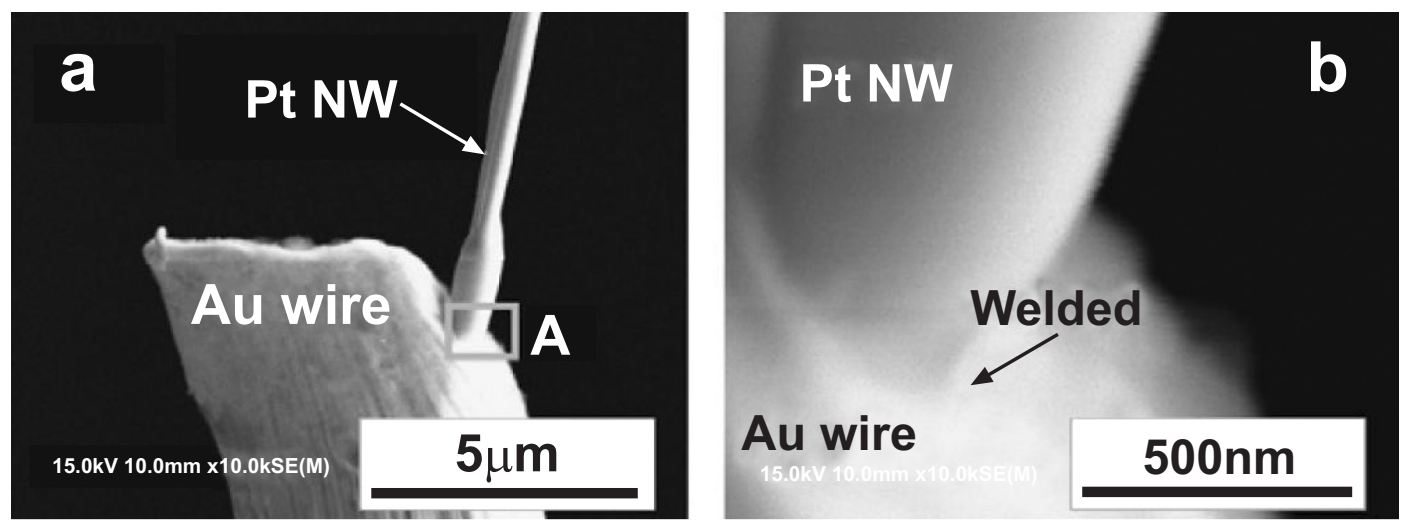

Fig. (10). SEM micrographs of welded joint between Pt nanowire and thin Au wire. A magnified view of point A in (a) is shown in (b) [24].

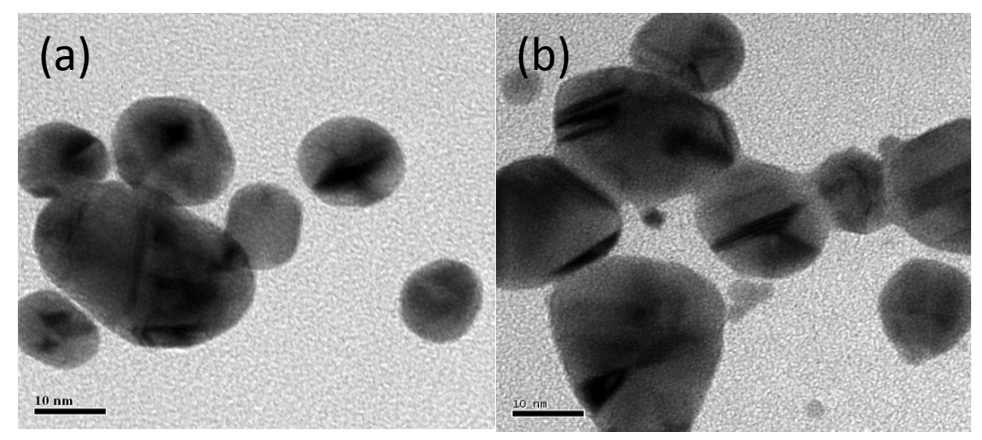

Fig. (11). TEM images of Au nanoparticles (a) before and (b) after femtosecond laser nanowelding [22].

dispersed in a carrier solution, onto substrates in one step with programming control. This technology can reduce the time, cost, and space consumed in mass production, and will merge well with current microelectronics manufacturing technologies. It is promising for incorporation into nanojoining processes such as diffusion nanobonding and nanosoldering/nanobrazing. For example, $\mathrm{Au}, \mathrm{Ag}$ and $\mathrm{Cu}$ nanoparticles, having potential to be used for microelectronics, can be printed and then sintered by various heating methods such as furnace [46], laser [47], and microwave [48]. Fig. (14) shows how a conductor pattern can be produced by laser sintering of inkjet-printed metallic nanoparticles on polymer substrate, in which unsintered nanoparticles are just simply washed away in an organic solvent [47].

\section{SUMMARY AND OUTLOOK}

As the size of building blocks continues to shrink into nanoscales, nanojoining is emerging only recently but developing rapidly, and is considered as one of the key technologies in industrially successful nano-devices and nano-systems. While new processes are certainly needed for this new area, some of the existing microjoining processes can be a source of inspiration when nanojoining processes are being developed. For example, various soldering processes and alloys, widely used in microelectronics interconnection and packaging, have been modified and 
further developed for the purpose of nanojoining [32]. Other examples include ultrasonic welding [14], cold welding [16],
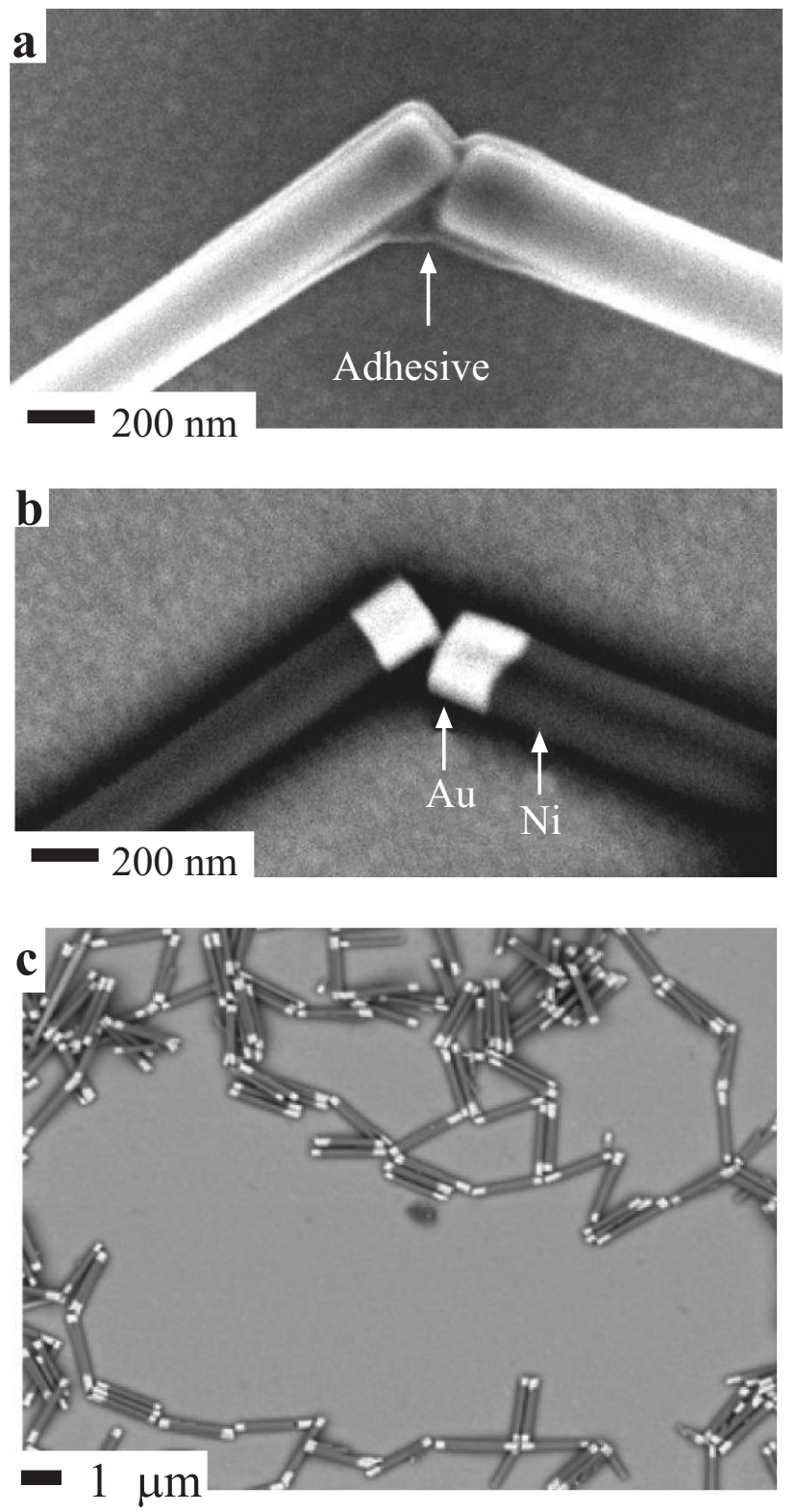

Fig. (12). (a) secondary electron SEM image showing the adhesive and rods, and backscattered electron SEM images (brighter regions correspond to materials with higher atomic numbers) showing (b) the $\mathrm{Au}$ segments appear brighter than the Ni parts (the polymer is not visible) and (c) 2D networks formed using the Au-Ni-Au rods [25].

laser welding [22], resistance welding [24], just to name a few. Focused electron beam, widely used in micro- and macro-joining [1] and already in joining of CNTs [10], should be suitable to join metallic nanomaterials. Similarly, focused ion beam, which is a relatively new energy source but has been utilized in trials of making nanojunctions between GaN nanowires [49], is promising in joining other nanomaterials. As new and innovative nanojoining processes continue to emerge, understanding will grow as to whether the traditional classification, in terms of solid-state bonding, fusion welding, brazing/soldering and adhesive bonding, can survive at the nanoscale.

Whether completely new or modified from microjoining (or even from macrojoining/regular joining processes), nanojoining processes face challenges because of everminiaturizing building blocks. For example, energy sources need to be increasingly accurately controlled to avoid excessive damage to bulk structures and properties in nanoscale components while robust and reliable joints are made [1, 22]. Novel devices or systems based on polymeric substrates and molecular components (such as photovoltaic, LCD and flexible microelectronics) require low temperature joining processes since these components cannot survive high temperature operations that are usually experienced in conventional joining. On the other hand, joints such as electrical interconnects, formed at low processing temperature, are frequently required to operate and survive at higher working temperatures, e.g., in automotive under-hood environments [11]. Moreover, as building blocks get smaller and smaller, e.g., to a size less than $100 \mathrm{~nm}$, surface forces may become dominant relative to gravitation forces, which could make the joining operation very difficult, e.g., even to release the components from the handling tools [42]. It is also difficult, or at least not economical, to manipulate most nanoscale components with current existing robotic techniques and assembly lines [50]. Developing appropriate assembly and nanojoining strategies is vital for commercially successful applications of nanodevices and nanosystems, such as micro-electro-mechanical systems (MEMS) and nano-electro-mechanical systems (NEMS). It is also expected that underlying physical and chemical phenomena may differ in many nanojoining processes even though they share technological resemblance to their counterparts in micro- and macro-joining. For example, it has been reported that transport mechanisms switch half-way through the sintering process of metallic nanoparticles, e.g., from surface diffusion to grain boundary diffusion [51].

\section{(A)}

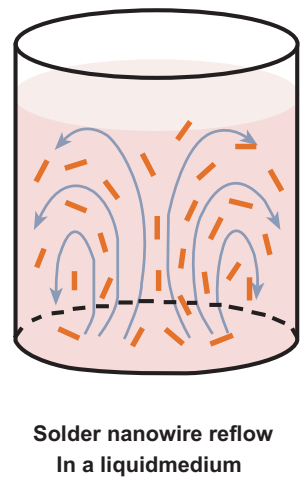

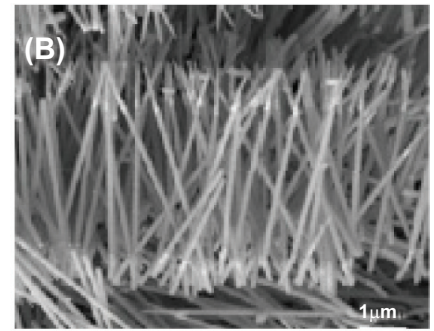

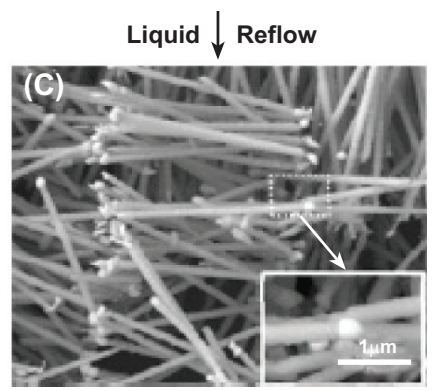

Fig. (13). Reflow soldering of magnetically self-organized Sn-Au$\mathrm{Ni}-\mathrm{Au}-\mathrm{Sn}$ multisegmented nanowires: (a) schematic of magnetically orientated solder nanowires in a liquid medium (b) nanowires before solder reflow (c) reflow soldered nanowires. Inset is a solder joint between two nanowires [17]. 

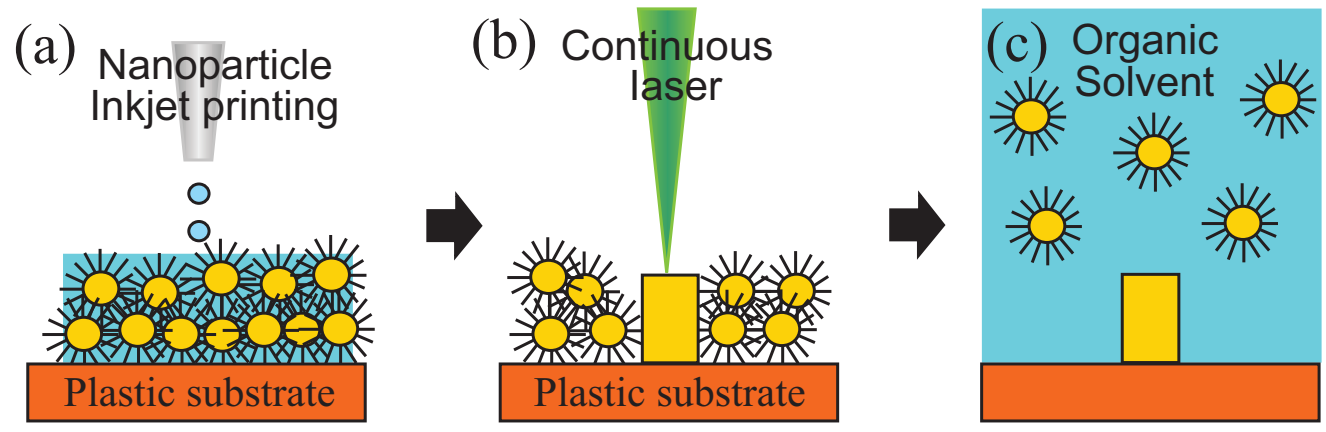

Fig. (14). Schematic showing laser sintering of inkjet-printed metallic nanoparticles on polymer substrate to form conductor patterns. Unsintered nanoparticles are simply washed away in an organic solvent [48].

While nanojoining technology is still in its infancy, most nanojoining work is on technological aspects, especially on developing new and modified processes to provide solutions for functional nanodevices and nanosystems. Work is, however, urgently needed on fundamental aspects of nanojoining for the long term progress of this area. These include the mechanisms of joining at nanoscale, such as driving forces, oxide removal, the effect of surface roughness, the formation of chemical bonds and crystallographic orientations across the bondline, especially in dissimilar material combinations, and effects of joining on the functionality, whether physical, chemical or mechanical, of nanoscale building blocks.

\section{REFERENCES}

[1] Zhou Y. Microjoining and nanojoining. Cambridge, England: Woodhead Publishing Ltd. CRC Press 2008.

[2] Cullison A. Welding: A heavyweight in a miniature world. Welding J 1996; 75: 29-34.

[3] Anderson O L, Christensen H, Andreatch P. Technique for connecting electrical leads to semiconductors. J Appl Phys 1957; 28: 92.

[4] Harman GG. Wire bonding in microelectronics-materials, processes, reliability and yield. $2^{\text {nd }}$ ed. New York: McGraw-Hill 1997.

[5] Stohr J A, Eriola J. Vacuum welding of metals. Welding and metal fabrication. 1958.

[6] Menz W, Mohr J, Paul O. Microsystem technology. Wiley-VCH 2001.

[7] Messler RW, Jr. Principles of welding: processes, physics, chemistry and metallurgy. New York: John Wiley \& Sons 1999.

[8] GÖsele U, Stenzel H, Martini T, Steinkirchner J, Conrad D, Scheerschmidt K. Self-propagating room temperature silicon wafer bonding in ultrahigh vacuum. Appl Phys Lett 1995; 67: 3614-6.

[9] Fukumoto S, Zhou Y. Mechanism of resistance microwelding of crossed fine nickel wires. Metall Mater Trans A 2004; 35A: 316576.

[10] Terrones M, Banhart F, Grobert N, Charlier JC, Terrones H, Ajayan PM. Molecular junctions by joining single-walled carbon nanotubes. Phys Rev Lett 2002; 89: 075505-1-4.

[11] Dilthey U, Dorfmueller T. Micro electron beam welding. Microsys Technol 2006; 12: 626-31.

[12] Hu A, Guo JY, Alarifi H, et al. Low temperature sintering of Ag nanoparticles for flexible electronics packaging. Appl Phys Lett 2010; 97(15): 153117-3.

[13] Ararifi A, Hu A, Yavuz M, Zhou Y. Silver nanoparticle paste for low-temperature bonding of copper. J Elec Mater 2011; In press.

[14] Chen C, Yan L, Kong E, Zhang Y. Ultrasonic nanowelding of carbon nanotubes to metal electrodes. Nanotechnology 2006; 17 : 2192-7.

[15] Chen C, Lu Y, Kong E S, Zhang Y, Lee S T. Nanowelded carbonnanotube-based solar microcells. Small 2008; 4: 1313-8.

[16] Lu Y, Huang J Y, Wang C, Sun S, Lou J. Cold welding of ultrathin gold nanowires. Nature Nanotechnol 2010; 5: 218-24.
[17] Gao F, Mukherjee S, Cui Q, Gu Z. Synthesis, characterization, and thermal properties of nanoscale lead-free solders on multisegmented metal nanowires. J Phys Chem C 2009; 113: 954652.

[18] Peng Y, Cullis T, Inkson B. Bottom-up nanoconstruction by the welding of individual metallic nanoobjectives using nanoscale solder. Nano Lett 2009; 9: 91-6.

[19] Wu W, Hu A, Li X, et al. Vacuum brazing of carbon nanotube bundles. Mater Lett 2008; 62: 4486-8.

[20] Zhou Y, Hu A, Khan MI, Wu W, Tam B, Yavuz M. Recent progress in micro and nano-joining. J Phys Conf Ser 2009; 165: 012012A.

[21] Mafune F, Kohno J, Takeda Y, Kondow T. Nanoscale soldering of metal nanoparticles for construction of higher-order structures. J Am Chem Soc 2003; 125: 1686-7.

[22] $\mathrm{Hu}$ A, Panda S K, Khan M I, Zhou Y. Laser welding, microwelding, nanowelding and nanoprocessing. Chin $\mathrm{J}$ Lasers 2009; 36: 3149-59.

[23] Kim S, Jang D. Laser-Induced nanowelding of gold nanoparticles. Appl Phys Lett 2005; 86: 033112-1-3.

[24] Tohmyoh H, Imazumi T, Hayashi H, Saka M. Welding of pt nanowires by joule heating. Scripta Mater 2007; 57: 953-6.

[25] Gu Z, Chen Y, Gracias DH. Surface tension driven self-assembly of bundles and networks of $200 \mathrm{~nm}$ diameter rods using a polymerizable adhesive. Langmuir 2004; 20: 11308-11.

[26] Hua W, Xiao S, Yang J, Zhang Z. Melting evolution and diffusion behavior of vanadium nanoparticles. Eur Phys J B 2005; 45: 54754.

[27] Wakuda D, Hatamura M, Suganuma K. Novel method for room temperature sintering of $\mathrm{Ag}$ nanoparticle paste in air. Chem Phys Lett 2007; 441: 305-8.

[28] Ide E, Angata S, Hirose A, Kobayashi K F. Metal-metal bonding process using Ag metallo-organic nanoparticles. Acta Mater 2003; 53: 2385-93.

[29] Tatsumi H, Akada Y, Yamaguchi T, Hirose A. Sintering mechanism of composite $\mathrm{Ag}$ nanoparticles and its application to bonding process -Effects of $\mathrm{Ag}_{2} \mathrm{CO}_{3}$ contents on bondability to $\mathrm{Cu}$. Adv Mater Res 2007; 26-28: 499-502.

[30] Morita T, Yasuda Y, Ide E, Akada Y, Hirose A. Bonding technique using micro-scaled silver-oxide particles for in situ formation of silver nanoparticles. Mater Trans 2008; 49: 2875-80.

[31] ASM Handbook, Volume 6: Welding, brazing, and soldering 1993.

[32] Cui Q, Gao F, Mukherjee S, Gu Z. Joining and interconnect formation of nanwires and carbon nantubes for nanoelectronics and nanosystems. Small 2009; 5: 1246-57.

[33] Girit C, Zetti A. Soldering to single atomic Layer. Appl Phys Lett 2007; 91: 193512-1-3.

[34] Ye H, Gu Z, Yu T, Gracias DH. Integrating nanowires with substrates using directed assembly and nanoscale soldering. IEEE Trans Nanotechnol 2006; 5: 62-6.

[35] Gu Z, Ye H, Smirnova D, Small D, Gracias DH. Reflow and electrical characteristics of nanoscale solder. Small 2006; 2: 225-9.

[36] Gao F, Gu Z. Nano-soldering of magnetically aligned threedimensional nanowire networks. Nanotech 2010; 21: 115604-11.

[37] Tohmyoh H, Fukui S. Self completed joule heat welding of ultrathin pt wires. Phys Rev B 2009; 80: 155403-1-7.

[38] Wu Y, Yang P. Melting and welding semiconductor nanowires in nanotubes. Adv Mater 2001; 13: 520-3. 
[39] Von der Linde D, Sokolowski-Tinten K, Biakowski J. Laser-solid interaction in the femtosecond time regime. Appl Surf Sci 1997; 109/110: 1-10.

[40] $\mathrm{Hu}$ A, Rybachuk M, Lu Q.-B, Duley WW. Low temperature sintering of $\mathrm{Ag}$ nanoparticles for flexible electronics packaging. Appl Phys Lett 2007; 91: 1319061-3.

[41] Whitesides GM, Grzybowski B. Self-Assembly at all scale. Science 2002; 295: 2418-21.

[42] Zheng W, Jacobs HO. Self-assembly process to integrate and connect semiconductor dies on surfaces with single-angular orientation and contact-pad registration. Adv Mater 2006; 18: 1387-91.

[43] Srinivasan U, Helmbrecht MA, Rembe C, Muller R, Howe R T. Fluidic self-assembly of micromirrors onto microactuators using capillary forces. IEEE Select Topic Quant Electron 2002; 8: 4-11.

[44] Gracias DH, Tien J, Breen TL, Hsu C, Whitesides G. Forming eelctrical networks in three dimensions by self-assembly. Science 2000; 18: 1170-2.

[45] Ding T, Song K, Clays K, Tung C. Fabrication of 3D Photonic crystals of ellipsoids: convection self-assembly in magnetic field. Adv Mater 2009; 21: 1936-40.
[46] Lee Y, Choi J, Lee K J, Stott N E, Kim D. Large-scale synthesis of copper nanoparticles by chemically controlled reduction for applications of inkjet-printed electronics. Nanotechnol 2008; 19: $415604-12$.

[47] Ko SH, Pan H, Grigoropoulos CP, Luscombe CK, Frechet JM, Poulikakos D. All-inkjet-printed flexible electronics fabrication on a polymer substrate by low-temperature high-resolution selective laser sintering of metal nanoparticles. Nanotechnol 2007; 18: 345202-10.

[48] Perelaer J, de Gans B, Schubert US. Ink-jet printing and microwave sintering of conductive silver tracks. Adv Mater 2006; 18: 2101-4.

[49] Dharam S, Lu CY, Wu CT, et al. Focused ion beam induced nanojunction and defect doping as a building block for nanoscale electronics in gan nanowires. J Phys Chem C 2010; 114: 15260-5.

[50] Zheng W, Buhlmann P, Jacobs HO. Sequential shape-and-solderdirected self-assembly of functional microsystems. PNAS 2004; 101: 12814-7.

[51] Ding L, Davidchack R L, J. Pan J. A molecular dynamics study of sintering between nanoparticles. Comput Mater Sci 2009; 45: 24756.

(C) Zhou and Hu; Licensee Bentham Open.

This is an open access article licensed under the terms of the Creative Commons Attribution Non-Commercial License (http://creativecommons.org/licenses/ by$\mathrm{nc} / 3.0 /$ ) which permits unrestricted, non-commercial use, distribution and reproduction in any medium, provided the work is properly cited. 